\title{
Sensorless Control of IPMSM Dispensing with Rotor Polarity Detecting
}

\author{
Dian-sheng SUN ${ }^{1,2, a, *}$, Yue-jin ZHANG ${ }^{1, b}$ \\ ${ }^{1}$ School of Mechatronic Engineering and Automation, Shanghai University, Shanghai, China \\ ${ }^{2}$ College of electrical Engineering, Binzhou University, Binzhou, China \\ a sds10001@163.com, ${ }^{\text {z } z h a n g y j @ m a i l . s h u . e d u . c n ~}$
}

\begin{abstract}
This paper proposes a new starting and low speed control method of sensorless control for permanent synchronous motor (PMSM) based on high frequency rotating voltage signal injection. This method can control the motor starting from standstill and running in low speed no need to distinguish the polarity of the rotor. The method include two rotor position estimating mode: open-loop estimating mode using in starting up status, and the closed-loop estimating mode using in low speed running status. The traditional sensorless drive scheme using high frequency rotating voltage injection can estimate the position of the rotor but can not distinguish the polarity, so the extra complex algorithm to distinguish the rotor position is often required. The proposed open-loop estimating mode of rotor position can solve this problem. When the rotor starting from standstill, if the estimated polarity of the rotor is not the truth, the pole $\mathrm{N}$ of the rotor can automatically adjust to the estimated position through a initial and short reverse rotation. This method based on saliency of the rotor but has no sensitivity to the armature resistance and inductance. The experiment results show the feasibility and effectiveness of the proposed method in a wide speed range including standstill.
\end{abstract}

Keywords: high-frequency signal injection; sensorless control; permanent magnet synchronous motor (PMSM); Dispensing with Rotor Polarity Detecting

\section{Introduction}

Permanent magnet synchronous motors (PMSM) with salient magnetic poles are used in many applications with advantages as high power density, high efficiency and fast dynamics ${ }^{[1]}$. The vector control of the PMSM can achieve high control performance but it requires the rotor angular position. Usually, a mechanical sensor such as an encoder is used to obtain the rotor angular position but it increases the size of the motor and the cost of the system.

The sensorless control of PMSM has been a challenging subject of interest to researchers for the last decade ${ }^{[2-6]}$. Many sensorless control techniques are not suitable for low speed and standstill condition such as back emf and sliding mode observer measurement. The high frequency carrier-signal injection method can work even in the low-speed and standstill condition and have no sensitivity to the armature resistance of the motor. However, the traditional high frequency carrier-signal injection method also has a problem that the rotor position (rotor direction) can be estimated but the polarity of the magnetic poles can not be distinguished. When the rotor starting from standstill, if the estimated magnetic polarity of the rotor is not the truth, the motor can not normally start up. In paper [2], the polarity-dependent d-axis magnetic saturation was modeled as a function of current by a second-order Taylor series and the magnet polarity was determined from the second harmonic of the injected frequency. Since the second harmonic of the injected high frequency signal is difficult to measure, this method is not very practical. Many series voltage pulses having the same amplitude and different directions were injected into the motor in [3-4], and the polarity of the magnetic poles was determined by the comparison of the current generated. This method requires high demands on operation and long time to detect and compare the response current. On the other hand, if the motor can starting normally, the magnetic poles polarity test is not necessary.

This paper presents a new starting and low speed control method no need to distinguish the polarity of the magnetic poles. The rotor can adjust its position through a initial and short reverse 
rotation automatically at the motor starting moment. After the motor starting successfully, a phase-locked loop method is used to estimate the position of the rotor with the rotating high-frequency voltage signal injection. This method simple, reliable and has the practical value.

\section{Principle of Rotor Position Estimated Using High Frequency Rotating Voltage Signal Injection}

A high-frequency rotating voltage signal is injected into the two phase stationary frame as (1)

$$
\left[\begin{array}{l}
u_{i \beta} \\
u_{i \alpha}
\end{array}\right]=\left[\begin{array}{c}
U_{i} \sin \left(\frac{\pi}{2}+\omega_{i} t\right) \\
U_{i} \cos \left(\frac{\pi}{2}+\omega_{i} t\right)
\end{array}\right]
$$

Where $u_{i \alpha}, u_{i \beta}$ is the $\alpha, \beta$ axis component of high- frequency voltage respectively.

So the high frequency current can be obtained as (2) ${ }^{[5]}$

$$
\left[\begin{array}{c}
i_{i \beta} \\
i_{i \alpha}
\end{array}\right]=\left[\begin{array}{c}
I_{i p} \sin \left(\omega_{1} t\right)+I_{i n} \sin \left(2 \theta-\omega_{i} t\right) \\
I_{i p} \cos \left(\omega_{1} t\right)+I_{i n} \cos \left(2 \theta-\omega_{i} t\right)
\end{array}\right]
$$

where $\theta$ is the electrical rotor position and $I_{i p}=\frac{U_{i}}{\omega_{i}}\left[\frac{L}{L^{2}-\Delta L^{2}}\right], I_{i n}=\frac{U_{i}}{\omega_{i}}\left[\frac{\Delta L}{L^{2}-\Delta L^{2}}\right]$ with $L=\left(L_{d}+L_{q}\right) / 2$ and $\Delta L=\left(L_{q}-L_{d}\right) / 2$.

The open-loop estimating mode of rotor position is describe as follows. The current is detected and transformed into high frequency rotating negative frame $\left(-\omega_{i} t\right)$ firstly. After eliminating the high frequency positive sequence current by low pass filter, the $I_{\text {in }} \sin (2 \theta)$ and $I_{\text {in }} \cos (2 \theta)$ are obtained and the pre-estimated electrical rotor position $\hat{\theta}_{1}$ can be calculated as

$$
\hat{\theta}_{1}=(1 / 2) \cdot \operatorname{atan} 2 P I\left(I_{\text {in }} \sin (2 \theta), I_{\text {in }} \cos (2 \theta)\right)
$$

The value domain of the function atan $2 P I(\cdot, \cdot)$ is $[0,2 \pi)$. Figure 1 shows the rotor rotation position when the estimated polarity is true or not. If the estimated polarity of the rotor is true, the rotor rotates in the specified direction as case 1 , and the motor start normally. If the estimated polarity of the rotor is wrong, the system regard $\mathrm{S}$ pole as $\mathrm{N}$ pole, the rotor rotates in the opposite direction of specified as case 2.

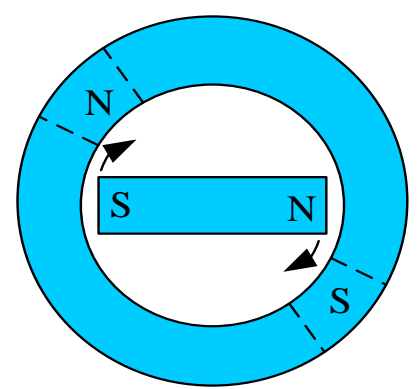

Case 1: the estimated polarity of the rotor is true
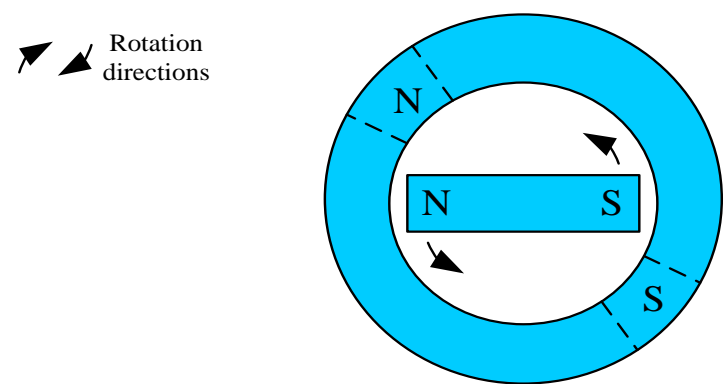

Case 2: the estimated polarity of the rotor is wrong

Fig. 1 The rotor rotation position when its estimated polarity is true or not

If the rotor rotates in the specified direction, the estimated electrical rotor position $\hat{\theta}$ can be obtained by frequency-division of $\hat{\theta}_{1}$ as (1) of Figure2. If the rotor rotates in the opposite direction of specified in the starting moment, the estimated and the real rotor position changes as (2) of Figure 2. So when the rotor rotates in the reverse direction, the $\hat{\theta}_{1}$ is used as the estimated electrical rotor position $\hat{\theta}$ without frequency-division. The pole $\mathrm{N}$ of the rotor can automatically adjust to the estimated position through a initial and short reverse rotation as shown in (2) of Figure 2. When the estimated value of the rotor position adjust to the real value along with the reverse rotation of the rotor, the rotor can rotates in the specified direction automatically. The rotation direction can be identified by the derivative of $\hat{\theta}_{1}$.

Figure 3 shows the simplified block diagram of proposed open-loop rotor position estimating structure at the time of starting. 

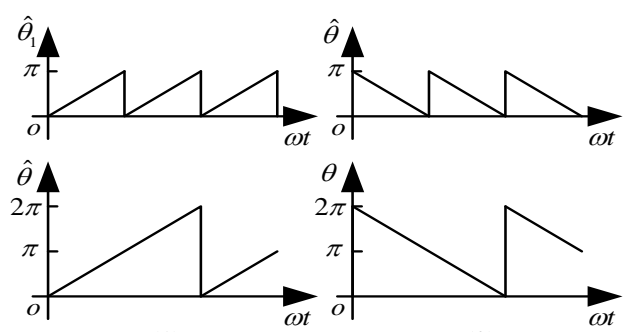

(1)

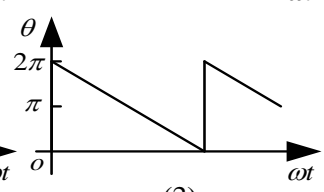

(2)

Fig.2 The estimated rotor position when its polarity is true or not

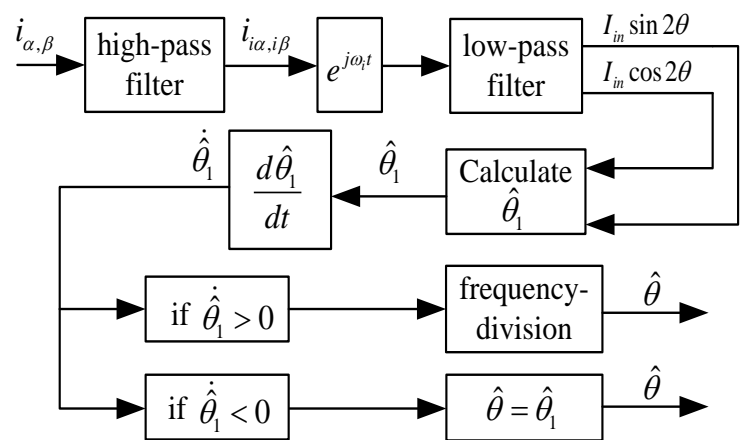

Fig.3 The proposed open-loop rotor position estimation structure at the time of starting

The open-loop estimating mode can realize the starting of the motor, but its reliability is not very high. The close-loop estimating mode can be used after the motor starting. The close-loop estimating mode of rotor position is describe as follows. Set up a formula as (4)

$$
i_{i \beta} \cos \left(2 \hat{\theta}-\omega_{i} t\right)-i_{i \alpha} \sin \left(2 \hat{\theta}-\omega_{i} t\right)
$$

and then (5) is obtained

$$
-I_{i p} \sin \left[2\left(\hat{\theta}-\omega_{i} t\right)\right]-I_{\text {in }} \sin [2(\hat{\theta}-\theta)]
$$

Then we can eliminate the high frequency term by a low pass filter and get $e_{\theta}=\theta-\hat{\theta} \approx-(1 / 2) \sin [2(\hat{\theta}-\theta)]$. when the difference between $\hat{\theta}$ and $\theta$ is very small. The $\hat{\theta}$ is directly estimated by a phase-locked loop as Figure 4.

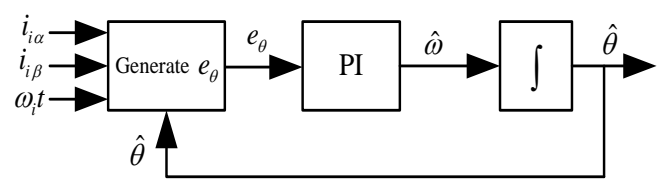

Fig.4 phase-locked loop to estimate $\hat{\theta}$

\section{Experiment Results and Its Analysis}

The proposed sensorless control method without rotor polarity detection was tested on a DSP controlled IPMSM. The test machine parameters were as follows: phase resistance $R=4.6 \Omega$, $L_{d}=6 \mathrm{mH}, L_{q}=12 \mathrm{mH}$. The poles is 10 , the rated speed is $2000 \mathrm{rpm}$ and the rated power is $1000 \mathrm{~W}$.

Measured high frequency component of current is shown in Figure 5. Figure 6 shows the measured $I_{\text {in }} \sin 2 \theta$ and $I_{\text {in }} \cos 2 \theta$.

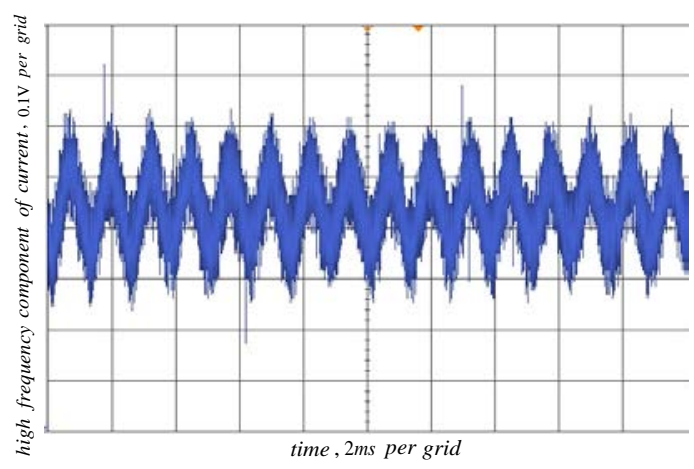

Fig.5 Measured high frequency component of current

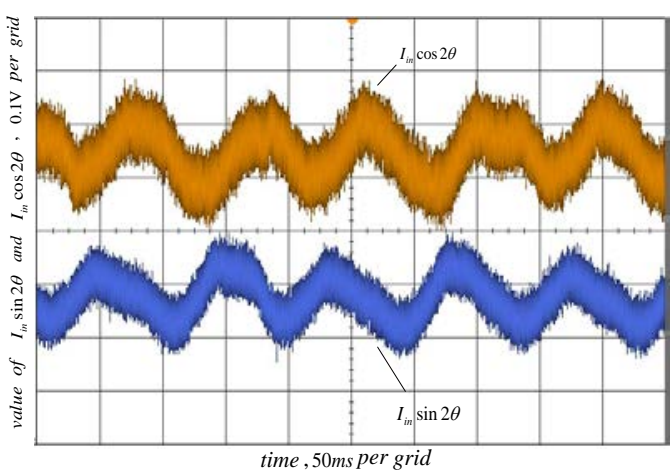

Fig.6 Measured $I_{\text {in }} \sin 2 \theta$ and $I_{\text {in }} \cos 2 \theta$

Figure 7 shows the estimated and measured electrical rotor position in starting up status. The (1) of Figure 7 shows the estimated polarity of the rotor is true and the rotor rotates in the setting direction directly. The (2) of Figure 7 shows the estimated polarity of the rotor is not true, the rotor rotates in the reverse direction firstly to adjust to the estimated position and then rotates in the setting direction. 


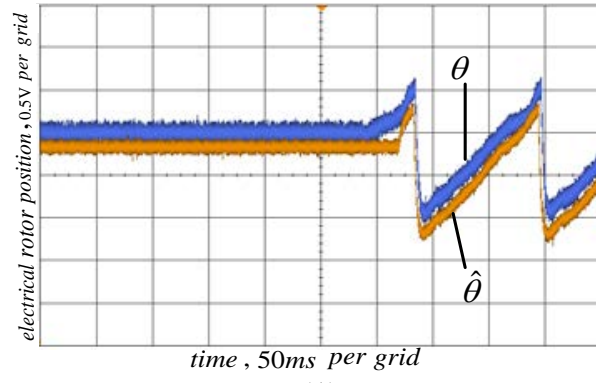

(1)

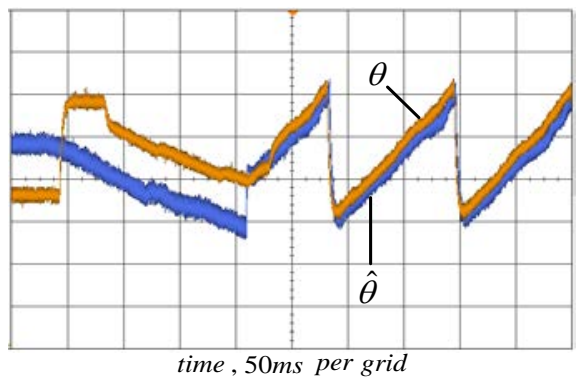

(2)

Fig.7 The estimate and measured value of electrical rotor position in starting up status

The estimated and measured electrical rotor position obtained using close-loop estimating mode is shown in figure 8.

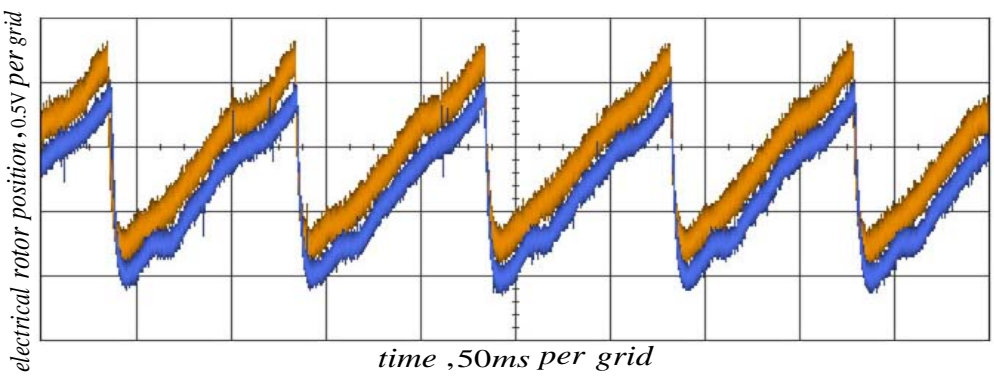

Fig.8 The estimate and measured value of electrical rotor position using close-loop estimating mode

\section{Conclusions}

A novel algorithm of sensorless controlling for permanent synchronous motor with salient magnet poles was presented. The rotor angular position estimated method based on high frequency rotating voltage signal injection can control the motor starting from standstill and running in low speed no need to distinguish the polarity of the rotor magnet poles. The open-loop estimating mode is used to realize the starting of the motor, and the close-loop estimating mode is used when the motor run in low speed after starting. The rotor position estimated value is obtained by phase-locked loop in the close-loop estimating mode. The experiment results show the feasibility and effectiveness of the proposed method in low speed and standstill.

\section{References}

[1] CHI W C, CHENG M Y. Implementation of a sliding-mode-based position sensorless drive for high-speed micro permanent-magnet synchronous motors[J]. ISA Trans, 2014, 53(2): 444-453.

[2] Hyunbae Kim, Kum-Kang Huh, Robert D. Lorenz, Thomas M. Jahns, A Novel Method for Initial Rotor Position Estimation for IPM Synchronous Machine Drives[J]. IEEE Trans Ind Appl, 2004,40(10): 1369-1378.

[3] CUPERTINO F, PELLEGRINO G, GIANGRANDE P, MEMBER S, SALVATORE L. Sensorless position control of permanent-magnet motors with pulsating current injection and compensation of motor end effects [J]. IEEE Trans Ind Appl, 2011, 47(3): 1371-1379.

[4] Wang Zihui, Lu Kaiyuan, Ye Yunyue. Initial position estimation method for permanent magnet synchronous motor based on improved pulse voltage injection[J]. Proceedings of the CSEE, 2011, 31(36): 95-101(in Chinese).

[5] RACA D, GARCÍA P, REIGOSA D D, BRIZ F, LORENZ R D.Carrier-signal selection for sensorless control of PM synchronous machines at zero and very low speeds [J]. IEEE Trans Ind Appl, 2010, 46(1): 167-178.

[6] JH Jang, SK Sul, JI Ha. Sensorless drive of surface mounted permanent-magnet motor by high-frequency signal injection based on magnetic saliency[J]. IEEE Trans on IA, 2003, 39 (4):1031- 1039. 